Kansas State University Libraries

New Prairie Press

\title{
NONLINEAR REGRESSION FOR SPLIT PLOT EXPERIMENTS
}

Marcia L. Gumpertz

John O. Rawlings

Follow this and additional works at: https://newprairiepress.org/agstatconference

Part of the Agriculture Commons, and the Applied Statistics Commons

\section{(c) $(1) \ominus$}

This work is licensed under a Creative Commons Attribution-Noncommercial-No Derivative Works 4.0 License.

\section{Recommended Citation}

Gumpertz, Marcia L. and Rawlings, John O. (1990). "NONLINEAR REGRESSION FOR SPLIT PLOT

EXPERIMENTS," Conference on Applied Statistics in Agriculture. https://doi.org/10.4148/2475-7772.1441

This is brought to you for free and open access by the Conferences at New Prairie Press. It has been accepted for inclusion in Conference on Applied Statistics in Agriculture by an authorized administrator of New Prairie Press. For more information, please contact cads@k-state.edu. 


\title{
NONLINEAR REGRESSION FOR SPLIT PLOT EXPERIMENTS
}

\author{
Marcia L. Gumpertz and John O. Rawlings \\ North Carolina State University
}

\section{ABSTRACT}

Split plot experimental designs are common in studies of the effects of air pollutants on crop yields. Nonlinear functions such the Weibull function have been used extensively to model the effect of ozone exposure on yield of several crop species. The usual nonlinear regression model, which assumes independent errors, is not appropriate for data from nested or split plot designs in which there is more than one source of random variation. The nonlinear model with variance components combines a nonlinear model for the mean with additive random effects to describe the covariance structure. We propose an estimated generalized least squares (EGLS) method of estimation for this model. The variance components are estimated two ways: by analysis of variance, and by an approximate MINQUE method. These methods are demonstrated and compared with results from ordinary nonlinear least squares for data from the National Crop Loss Assessment Network (NCLAN) program regarding the effects of ozone on soybeans. In this example all methods give similar point estimates of the parameters of the Weibull function. The advantage of estimated generalized least squares is that it produces proper estimates of the variances of the parameters and of estimated yields, which take the covariance structure into account. A computer program that fits the nonlinear model with variance components by the EGLS method is available from the authors.

KEYWORDS: Random effects; Variance components; Mixed models

\section{INTRODUCTION}

Split plot experimental designs are often used in studies of the effects of air pollutants on crop yields. For example, in the National Crop Loss Assessment Network (NCLAN) program the effects of ozone exposure on yield of soybean were studied at Beltsville, Maryland in 1983 (Heggestad et al 1985). In this experiment ozone was dispensed in open top field chambers. There were ten treatments, consisting of combinations of five ozone exposure levels: charcoal filtered $(\mathrm{CF})$, nonfiltered $(\mathrm{NF}), \mathrm{NF}+.03 \mathrm{ppm}, \mathrm{NF}+.06 \mathrm{ppm}$, and NF +.09 ppm; and two watering regimes: well watered and water stressed. Thirty chambers were arranged in three randomized blocks with 10 chambers per block. Within each chamber two cultivars (Corsoy and Williams) of soybeans were grown. In this experiment ozone level and watering regime are whole plot treatments and the cultivars are the split plot treatments.

One of the objectives of this type of experiment is to model the effect of ozone on yield to produce estimates of the relative yield loss due to increases or decreases in ozone exposure. The Weibull model has been found to be a flexible monotonically decreasing model that fits the data well for several crop species (Rawlings and Cure 1985). The usual nonlinear regression model assumes that all observations are taken independently. In the split plot situation the observations within a whole plot; i.e. within a chamber in this example, are expected to be correlated, violating the assumption of independent observations. In this situation a Weibull model for the mean with two random effects, one for the whole plot error and one for the subplot error, would be appropriate. This is a special case of a nonlinear model with variance components (also called a nonlinear model with random effects). This type of model consists of a nonlinear function for the mean and some additive random effects. It is similar to a mixed model in the analysis of variance, except that the function for the mean is allowed to be a nonlinear function.

In Section 2 we present the equation for the nonlinear model with variance components and give the Weibull model for the soybean data. The method of estimating the parameters 
called estimated generalized least squares (EGLS) is discussed in Section 3. Implementation of estimated generalized least squares requires that the variance components be estimated. Two methods of estimating the variance components are presented in Section 4. In the final section results for the soybean yield data using estimated generalized least squares are compared to the results from ordinary nonlinear least squares.

\section{NONLINEAR MODEL WITH VARIANCE COMPONENTS}

The nonlinear model with split plot errors is a special case of the nonlinear model with variance components. In this model there is a nonlinear function for the mean, $\underset{\sim}{\mathrm{g}}(\underset{\sim}{\mathbb{X}}, \underset{\sim}{,})$, and the random effects, such as whole plot errors, are added to the mean function. The model is:

where

$$
\underset{\sim}{\mathrm{y}}=\underset{\sim}{\mathrm{g}}(\underset{\sim}{\mathrm{X}}, \underset{\sim}{\theta})+\sum_{k=0}^{r} \mathrm{U}_{k} \mathrm{e}_{k}
$$

$g(\underset{\sim}{X}, \underset{\sim}{,})$ is the function for the mean,

$\mathrm{e}_{k}$ is the $k^{\text {th }}$ random effect, and

$\mathrm{U}_{k}$ contains indicator variables for the $k^{\text {th }}$ random effect.

We assume that the random effects are normally distributed and are independent of each other:

$$
\begin{aligned}
& \stackrel{\mathrm{e}}{\sim}_{k} \sim \mathrm{N}\left(0, \sigma_{k}^{2} \mathrm{I}\right) \\
& \stackrel{\mathrm{e}}{\sim}_{k}, \mathrm{e}_{j} \text { independent for } k \neq j .
\end{aligned}
$$

The following data requirements ensure that the parameters can be estimated:

1. $n \geq p+r+1$. The total number of observations must be at least as large as the number of parameters being estimated.

2. $\underset{\sim}{\mathrm{D}}(\underline{\theta})$ has full column rank, where $\underset{\sim}{\mathrm{D}}(\underline{\underline{\theta}})=\left[\frac{\partial \mathrm{g}(\theta)}{\partial \theta_{1}}, \frac{\partial \mathrm{g}(\theta)}{\partial \theta_{2}}, \ldots, \frac{\partial \mathrm{g}(\theta)}{\partial \theta_{p}}\right]$. In nonlinear regression the derivative matrix $\mathrm{D}(\underset{\sim}{\theta})$ corresponds to the design matrix in linear regression. This is similar to the requirement that the design matrix have full column rank.

3. Rank of $\left.[\underset{\sim}{D}(\underset{\theta}{\theta}) \mid \underbrace{}_{k}]_{k}\right]>$ rank of $\underset{\sim}{D}(\theta)$. The indicator variables for the random effects must not be linear combinations of the columns of $\underset{\sim}{\mathrm{D}}(\theta)$.

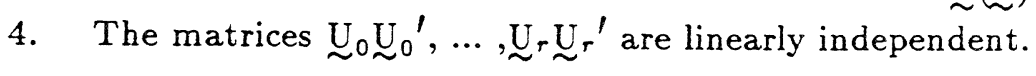

For the soybean experiment described in section 1 the mean function, $g(\underset{\sim}{X}, \theta)$, is a Weibull function relating $y=$ soybean yield $(\mathrm{kg} / \mathrm{ha}$ ) to $x=$ ozone exposure (ppm). The basic Weibull model has been modified to accomodate block, moisture, and cultivar effects and the whole plot chamber-to-chamber variation is added to the Weibull function. The model is:

$$
y_{i j k l}=\left(\alpha+B_{i}+M_{j}+C_{l}+(M C)_{j l}\right) \cdot \mathrm{e}^{-\left(\frac{x_{i j k l}}{\omega_{j}}\right)^{\lambda}}+\delta_{i j k}+\epsilon_{i j k l},
$$

where $i=1,2,3$ blocks; $j=1,2$ moisture regimes; $k=1, \ldots, 5$ ozone levels; and $l=1,2$ cultivars. In this model $\alpha$ gives the maximum yield for Williams soybeans in the well watered chambers. The parameters $\mathrm{B}_{i}, \mathrm{M}_{j}, \mathrm{C}_{l}$, and (MC) ${ }_{j l}$ adjust $\alpha$ for block, moisture, and cultivar effects. The Weibull parameter $\omega_{j}$ gives the ozone level at which yield is reduced $63 \%$, and $\lambda_{j}$ is a shape parameter for the Weibull function. The whole plot error is labeled $\delta_{i j k}$, and the within-chamber error is labeled $\epsilon_{i j k l}$. This model has separate parameters $\omega_{j}$ and $\lambda_{j}$ for each moisture regime, but not for the two cultivars. Within each moisture treatment the shape of 
the curve appears similar for the two cultivars but the maximum yield was higher for Williams than for Corsoy (Fig. 1). A more flexible model, with separate $\omega_{j l}$ and $\lambda_{j l}$ parameters for each moisture $\mathrm{X}$ cultivar combination could also have been fit. A model selection procedure is described in Gumpertz and Rawlings (1990) in detail.

\section{GENERALIZED LEAST SQUARES ESTIMATION OF PARAMETERS}

In ordinary nonlinear least squares (OLS) the parameters are estimated by minimizing the sum of the squared residuals:

$$
(\underset{\sim}{\mathrm{y}}-\underset{\sim}{\mathrm{g}}(\underset{\sim}{\mathrm{X}}, \underset{\sim}{\theta}))^{\prime}(\underset{\sim}{\mathrm{y}}-\underset{\sim}{\mathrm{g}}(\underset{\sim}{\mathrm{X}}, \underset{\sim}{\theta})) \text {. }
$$

This method gives equal weight to all observations and is the method of choice if the observations are all independent and the errors arise from a common distribution. These conditions are often satisfied if the data come from a completely randomized design.

If the errors do not all have the same variance or if they are not independent, then the method of generalized least squares (GLS) may be preferred. This method is similar to ordinary least squares except that the parameters are estimated by minimizing a sum of squares of weighted residuals,

$$
(\underset{\sim}{\mathrm{y}}-\underset{\sim}{\mathrm{g}}(\underset{\sim}{\mathrm{X}}, \underset{\sim}{\theta}))^{\prime} \underline{\sim}^{-1}(\mathrm{y}-\underset{\sim}{\mathrm{g}}(\underset{\sim}{\mathrm{X}}, \underset{\sim}{\theta})),
$$

where the matrix $\underset{\sim}{\mathrm{V}}$, the covariance matrix of $\underset{\sim}{\mathrm{y}}$, contains information about the relative weights to assign to the observations. In the split plot design of our example the observations within a chamber are correlated. The variance-covariance matrix of $\mathbf{y}$ is block diagonal with ijk $^{\text {th }}$ block:

$$
\mathrm{V}_{i j k}=\left[\begin{array}{cc}
\sigma_{\epsilon}^{2}+\sigma_{\delta}^{2} & \sigma_{\delta}^{2} \\
\sigma_{\delta}^{2} & \sigma_{\epsilon}^{2}+\sigma_{\delta}^{2}
\end{array}\right] .
$$

The observations do not all contribute independent information and so they are weighted accordingly.

Generalized least squares estimation can be implemented using the Gauss-Newton algorithm. The algorithm for minimizing the objective function

$$
(\underline{\sim}-\underset{\sim}{g}(\underset{\sim}{\mathrm{X}}, \underset{\sim}{\theta}))^{\prime} \underline{\sim}^{-1}(\underset{\sim}{\mathrm{y}}-\underset{\sim}{\mathrm{g}}(\underset{\sim}{\mathrm{X}}, \underset{\sim}{\theta}))
$$

has the following steps.

1. Supply an initial value, ${\underset{\sim}{0}}_{0}$, for $\underset{\sim}{\theta}$.

2. Premultiply both sides of the original model by $\mathrm{V}^{-1 / 2}$,

$$
\underline{\mathrm{V}}^{-1 / 2} \underset{\sim}{\mathrm{z}}={\underset{\sim}{\mathrm{V}}}^{-1 / 2} \underset{\sim}{\mathrm{g}}(\underset{\sim}{\mathrm{X}}, \theta)+\underset{\sim}{\mathrm{V}^{-1 / 2}} \sum_{k=0}^{r} \mathrm{U}_{k} \mathrm{e}_{k} .
$$

Substitute the first order Taylor series expansion of ${\underset{\sim}{V}}^{-1 / 2} \mathrm{~g}(\underset{\sim}{\mathrm{X}}, \underset{\sim}{\sim})$ around ${\underset{\sim}{0}}_{0}$ into the model for $\mathrm{V}^{-1 / 2} \mathrm{y}$ to get the following expression:

$$
{\underset{\sim}{\mathrm{V}}}^{-1 / 2}\left(\underset{\sim}{\mathrm{y}}-\underset{\sim}{\mathrm{g}}\left(\underset{\sim}{\mathrm{X}}, \underline{\sim}_{0}\right)\right) \doteq{\underset{\sim}{\mathrm{V}}}^{-1 / 2} \mathrm{D}\left(\underline{\theta}_{0}\right)\left(\underline{\sim}-\underline{\sim}_{0}\right)+{\underset{\sim}{\mathrm{V}}}^{-1 / 2} \sum_{k=0}^{r} \mathrm{U}_{k} \mathrm{e}_{k} .
$$


3. Solve for $\underset{\sim}{\theta}$ using OLS on ${\underset{\sim}{V}}^{-1 / 2}\left(\underset{\sim}{\mathrm{y}}-\underset{\sim}{\mathrm{g}}\left(\underset{\sim}{\mathrm{X}}, \theta_{0}\right)\right)$ :

$$
\stackrel{\hat{\theta}}{\sim}_{1}-\underline{\sim}_{0}=\left[\underset{\sim}{D}\left(\underline{\sim}_{0}\right)^{\prime} \underline{\sim}^{-1} \mathrm{D}\left(\underline{\sim}_{0}\right)\right]^{-1} \mathrm{D}\left(\underline{\theta}_{0}\right)^{\prime} \underline{\sim}^{-1}\left(\underset{\sim}{\mathrm{y}}-\underset{\sim}{\mathrm{g}}\left(\underset{\sim}{\mathrm{X}}, \underline{\sim}_{0}\right)\right) .
$$

\section{Iterate to convergence.}

In the nonlinear model with random effects the generalized least squares estimator has good statistical properties. Under certain conditions it is strongly consistent, asymptotically normal, and asymptotically efficient (Gumpertz and Pantula 1990). It is not feasible to use in actual practice, however, because the covariance matrix, $\underset{\sim}{\mathrm{V}}$, is unknown.

\section{VARIANCE COMPONENT ESTIMATION AND ESTIMATED GENERALIZED LEAST SQUARES}

In the context of analysis of variance, procedures for estimating variance components are simple if the data come from a designed experiment with no missing observations. In the split plot case, for example, the usual procedure is to compute the mean squares for the analysis of variance table, set the mean squared errors for whole plots and subplots, $\mathrm{MSE}_{a}$ and $\mathrm{MSE}_{b}$ respectively, equal to their expected values, and solve for $\sigma_{a}^{2}$ and $\sigma_{b}^{2}$.

When the data are unbalanced or when a linear model other than an analysis of variance model is used to fit the data, there is no unique unbiased estimator of the variance components. The method of minimum variance quadratic unbiased estimation (MIVQUE) is one method of obtaining unbiased estimates that is available for unbalanced linear mixed models. C. R. Rao (1972) showed that of all quadratic equations of $\underset{\sim}{y}$, the $r+1$ quadratic forms

$$
(\underset{\sim}{\mathrm{y}}-\underset{\sim}{\hat{\mathrm{y}}} \mathrm{GLS})^{\prime} \underline{\sim}^{-1}{\underset{\sim}{\mathrm{U}}}_{k} \mathrm{U}_{k}^{\prime} \underline{\sim}^{-1}\left(\underset{\sim}{\mathrm{y}}-{\underset{\sim}{\hat{y}}}_{G L S}\right), \quad k=0, \ldots r
$$

lead to the unbiased estimates of $\sigma_{k}^{2}$ with smallest variance. The MIVQUE procedure is to set these quadratic forms equal to their expected values and then solve for the $\sigma_{k}^{2}$. This procedure is similar to the analysis of variance method for balanced data except that the quadratic forms of $y$ that are equated to their expectations are not sums of squares from any traditional anẩysis of variance. When the data are balanced the MIVQUE estimates coincide with the analysis of variance estimates.

Variance component estimation by MIVQUE can also be viewed as a linear regression based on the residuals, $\mathrm{r}_{i j k l}=\mathrm{y}_{i j k l}-\hat{\mathrm{y}}_{\mathrm{GLS} i j k l}$, from the generalized least squares fit of the model. If we arrange all of the squared residuals, $r_{i j k l}^{2}$, and cross products of residuals, $\mathrm{r}_{i j k l} \mathrm{r}_{m n o p}$, in a vector, set them equal to their expectations, and solve for $\sigma_{a}^{2}$ and $\sigma_{b}^{2}$ by generalized least squares, the resulting variance component estimates are the MIVQUE estimates (Brown 1978).

Of course the covariance matrix, $\underset{\sim}{V}$, is unknown, so the MIVQUE equations cannot be solved exactly. What is done in practice is to use an initial guess for the $\underset{\sim}{\mathrm{V}}$ matrix (this procedure is called MINQUE) or to start with an initial guess and then iterate the procedure, updating the $\underset{\sim}{\mathrm{V}}$ matrix at each iteration (iterated MINQUE). For the soybean data an approximation to the iterated MINQUE method was used. In nonlinear regression the MIVQUE expression $(\underset{\sim}{\mathrm{y}}-\underset{\sim}{\hat{y}})^{\prime} \underline{\sim}^{-1}{\underset{\sim}{k}}_{\underset{\sim}{*}}^{U_{k}} \underline{\sim}^{-1}(\underset{\sim}{\mathrm{y}}-\underset{\sim}{\hat{y}})$ is not exactly a quadratic form of $\underset{\sim}{\mathrm{y}}$, so by definition the method is not exactly quadratic estimation. The residuals from the ordinary least squares fit, $y-\hat{y}$ OLS, were used in this expression. The initial values for the matrix $\hat{\sim}$ were obtained from a split plot analysis of variance. At every subsequent iteration the estimated covariance matrix, $\underset{\sim}{\hat{V}}$, was updated and the residual vector, $\underset{\sim}{\mathrm{v}}-\hat{\mathfrak{y}}_{\mathcal{L}}$ Ls , was updated using a linearizing approximation.

Once the variance components have been estimated, they can be substituted into the NewPrairiePress 
generalized least squares equation (3.2) for estimating the parameters of the mean model. The estimated generalized least squares algorithm has the following three steps:

1. Obtain OLS estimate of $\underset{\sim}{\theta}$.

2. Estimate variance components using the ANOVA approach or the MIVQUE approach.

3. Minimize $(\underset{\sim}{\mathrm{y}}-\underset{\sim}{\mathrm{g}}(\underset{\sim}{\mathrm{X}}, \underset{\sim}{\sim}))^{\prime}{\underset{\sim}{\mathrm{V}}}^{-1}(\underset{\sim}{\mathrm{y}}-\underset{\sim}{\mathrm{g}}(\underset{\sim}{\mathrm{X}}, \underset{\sim}{\theta}))$.

\section{EXAMPLE: EFFECTS OF OZONE ON SOYBEAN YIELD}

The NCLAN study of the effects of ozone exposure on soybean yields was designed as a balanced split plot experiment. The only imbalance occured because the observed ozone levels for each ozone treatment varied slightly from block to block. The first step in our analysis, therefore, was to compute the standard split plot analysis of variance given in Table 1, using the nominal ozone treatment levels instead of the actual observed ozone concentrations. The analysis of variance indicates that the effect of ozone concentration depends on whether the plots were well watered or water-stressed ( $F$ - test for ozone $x$ moisture interaction, $p$-value $<$ .0001 ). Yield decreases as ozone increases (Fig. 1), but the maximum yield is different for the two cultivars ( $p$-value $<.0001$ ), with the difference between the two cultivars depending on the moisture regime ( $\mathrm{p}$-value $=.02$ ).

The Weibull model given in equation (2.2) incorporates this information about cultivar and moisture level effects. There is a separate set of parameters for each moisture level and the maximum yield is allowed to vary depending on moisture level, cultivar, and block. The second step in the analysis was to fit the Weibull curve by the method of estimated generalized least squares using the SASRC ${ }^{1}$ macro program NLINVC (available from the authors). Tables 2 and 3 give estimates of the variance components and their standard errors computed three different ways: 1) analysis of variance method, 2) approximate iterated MINQUE (labelled NLINVC-MML in the table), and 3) approximate maximum likelihood (labelled NLINVC-ML in the table). The analysis of variance and the iterated MINQUE estimates of the variance components are similar. The iterated MINQUE estimates are slightly larger than the analysis of variance estimates, probably because the Weibull model does not fit the treatment means exactly; that is, there is some lack of fit in the Weibull model as compared to the analysis of variance model. The approximate maximum likelihood estimator of the variance components is asymptotically normal and efficient (Gumpertz and Pantula 1990) but the sample size of this experiment may not be large enough to warrent its use. The NLINVC-ML estimates of the variance components appear to be biased downward in this experiment, especially the estimate of $\sigma_{a}^{2}$. Consequently the NLINVC-ML variance component estimates were not used for any further analysis.

The parameters of the Weibull model estimated by ordinary least squares and estimated generalized least squares are reported in Table 4. The EGLS estimates were computed using both the analysis of variance and the iterated MINQUE estimates of the variance components. The two sets of EGLS estimates were identical, and the OLS estimates were very similar to the EGLS estimates. Under the assumptions of the nonlinear model with variance components (2.1), the asymptotic variance matrix of the ordinary least squares estimator is given by the expression

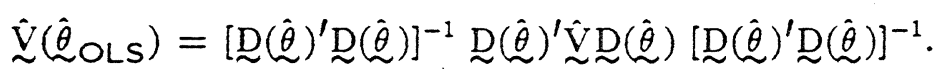

This is not the variance matrix printed out by standard nonlinear regression programs such as

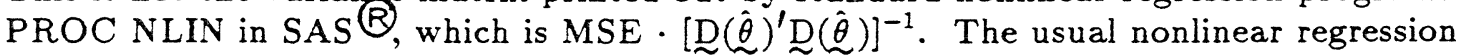
programs assume that there is only one source of random error, which is estimated by MSE. This estimate is intermediate between the two variance components required in a split plot

8000 .

${ }^{1} \mathrm{SAS}$ is a registered trademark of SAS Institute, Inc., Box 8000, Cary, NC 27512- 
experiment. Therefore, standard nonlinear regression programs will usually underestimate the variance of parameters that measure differences among whole plots and overestimate the variance of parameters that measure differences among subplots within a whole plot. The correct asymptotic standard errors computed by NLINVC and the incorrect standard errors from PROC NLIN are shown in Table 5. PROC NLIN underestimates the variance of all parameters having to do with block, moisture, and ozone effects and overestimates the variance of the parameters regarding cultivar effects.

When the appropriate standard errors are compared, the OLS and EGLS Weibull parameter estimates have very similar standard errors in the soybean experiment. Table 6 gives the standard errors of the parameter estimates computed by OLS, EGLS using analysis of variance to estimate the variance components, and EGLS using the iterated MINQUE estimates of the variance components. The first column of this table is the same as the second column of Table 5. The standard errors of the EGLS estimates are taken from the asymptotic variance matrix,

$$
\hat{\mathrm{V}}(\stackrel{\hat{\theta}}{\mathrm{EGLS}})=\left[\mathrm{D}(\hat{\hat{\theta}})^{\prime} \underline{\sim}^{-1} \mathrm{D}(\hat{\dot{\theta}})\right]^{-1}
$$

The standard errors of the OLS estimates were computed using equation (5.1) where $\hat{\mathrm{V}}$ is based on the iterated MINQUE variance component estimates. Therefore, the OLS standard errors are directly comparable to the standard errors of the EGLS estimates computed using the MINQUE variance components. Comparison of the first and third columns of Table 6 shows that for this particular data set the standard errors of the OLS and the EGLS estimates are very similar to each other. The standard errors for EGLS estimates computed using the ANOVA variance component estimates were smaller than the other two sets of estimates because the variance component estimates from the analysis of variance method were smaller than from iterated MINQUE. It appears that this difference is due to some lack of fit of the Weibull model. For the purpose of assessing the variability of the Weibull parameter estimates it is realistic to include the lack of fit, so the standard errors based on the MINQUE variance components are emphasized here.

\section{SUMMARY}

The nonlinear model with variance components (2.1) is appropriate for experiments with randomized block, split plot, and nested designs where the objective is to fit a nonlinear model. The data do not need to be balanced to apply the estimated generalized least squares method presented in this paper. In estimated generalized least squares the variance components are first estimated using the analysis of variance if the data are balanced, or using an approximate iterated MINQUE procedure when the data are unbalanced. There are several other methods of estimating the variance components that could be used; these two methods do not exhaust the possibilities. The estimated variance matrix is substituted into the generalized least squares estimating equations (3.2) to obtain the EGLS estimates of the parameters of the mean function. The estimated generalized least squares approach produces estimates that are more efficient than ordinary least squares when the sample size is large (Gumpertz and Pantula 1990).

When the sample size is small or moderate, ordinary least squares may give estimates that have standard errrors as small or smaller than estimated generalized least squares. In the example of Section 5 the variation among chambers, $\sigma_{a}^{2}$, was not estimated very precisely; the variance component estimate was not even twice its standard error (Tables 2 and 3 ). In this type of situation the uncertainty in $\hat{\sigma}_{a}^{2}$ contributes more to the EGLS parameter estimates than to the OLS parameter estimates and so OLS may be preferred. If the parameters are estimated by ordinary least squares, variance component estimates are still required in order to obtain correct asymptotic standard errors. Standard nonlinear regression programs do not produce the correct asymptotic standard errors for model (2.1). The correct standard errors, 
given by expression (5.1), take the covariance structure into account. In the soybean example ordinary least squares and estimated generalized least squares produced very similar point estimates and standard errors for the parameters of the Weibull function.

\section{ACKNOWLEDGMENTS}

Research partly supported by Interagency Agreement between the USEPA and the USDA: Interagency Agreement no. DW 12931347, and the Specific Cooperative Agreement no. 58-43YK-6-0041 between the USDA and the North Carolina Agriculture Research Service. The authors thank H.E. Heggestad for the use of the soybean data.

\section{REFERENCES}

Brown, K. G. 1978. Estimation of variance components using residuals. Journal of the American Statistical Association 73: 141-146.

Gumpertz, M. G. and S. G. Pantula. 1990. Nonlinear regression with variance components. Submitted to Journal of the American Statistical Association.

Gumpertz, M. G. and J. O. Rawlings. 1990. Nonlinear regression with variance components: modeling effects of ozone on crop yields. Submitted to Crop Science.

Heggestad, H. E., Gish, T. J., Lee, E. H., Bennett, J. H. and L. W. Douglass. 1985. Interaction of soil moisture stress and ambient ozone on growth and yields of soybeans. Phytopathology 75: 472-477.

Rao, C. R. 1972. Estimation of variance and covariance components in linear models. Journal of the American Statistical Association 67: 112-115.

Rawlings, J. O. and W. W. Cure. 1985. The Weibull function as a dose-response model to describe ozone effects on crop yields. Crop Science 25: 807-814.

Table 1. Analysis of Variance

\begin{tabular}{lccl} 
Source & df & Mean Square & P-Value \\
\hline Block & 2 & 58,600 & .65 \\
Ozone & 4 & $20,500,000$ & .0001 \\
Moisture & 1 & 159,000 & .29 \\
$O_{3} \times$ moisture & 4 & $1,420,000$ & .0001 \\
Error a & 18 & 302,000 & \\
Cultivar & 1 & $20,100,000$ & .0001 \\
Cultivar $\times$ moist. & 1 & 852,000 & .021 \\
Cultivar $\times O_{3}$ & 4 & 226,000 & .19 \\
Cult. $\times O_{3} \times$ moist. & 4 & 153,000 & .37 \\
Error $b$ & 20 & 135,000 & \\
\hline
\end{tabular}


Table 2. Estimates of Variance Components

\begin{tabular}{cccc} 
& & "MINQUE" & "ML" \\
Component & ANOVA & NLINVC-MML & NLINVC-ML \\
\hline$\sigma_{a}^{2}$ & 83503 & 97181 & 55972 \\
$\sigma_{b}^{2}$ & 134624 & 158033 & 147209 \\
\hline
\end{tabular}

Table 3. Standard Errors of Variance Components

"MINQUE" "ML"

\begin{tabular}{llcc} 
Component & ANOVA & NLINVC-MML & NLINVC-ML \\
\hline$\sigma_{a}^{2}$ & 54592 & 57295 & 33935 \\
$\sigma_{b}^{2}$ & 42572 & 42423 & 36592 \\
\hline
\end{tabular}

Table 4. Weibull Parameter Estimates

\begin{tabular}{cccc} 
& & EGLS & EGLS \\
Parameter & OLS & ANOVA & MINQUE \\
\hline$\alpha$ & 8036 & 8000 & 8000 \\
$B_{1}$ & -194 & -193 & -193 \\
$B_{2}$ & -14 & -15 & -15 \\
$C_{1}$ & -1971 & -1963 & -1963 \\
$M_{1}$ & -1005 & -1047 & -1047 \\
$(\mathrm{CM})_{11}$ & 759 & 763 & 763 \\
$\omega_{1}$ & .12 & .12 & .12 \\
$\lambda_{1}$ & 1.48 & 1.55 & 1.55 \\
$\omega_{2}$ & .092 & .092 & .092 \\
$\lambda_{2}$ & 1.92 & 1.92 & 1.92 \\
\hline
\end{tabular}


Table 5. Standard Errors of Parameter Estimates

Ordinary Least Squares

\begin{tabular}{ccc} 
Parameter & NLIN & NLINVC \\
\hline$\alpha$ & 361 & 418 \\
$\mathrm{~B}_{1}$ & 215 & 258 \\
$\mathrm{~B}_{2}$ & 216 & 259 \\
$\mathrm{C}_{1}$ & 264 & 222 \\
$\mathrm{M}_{1}$ & 576 & 672 \\
$(\mathrm{CM})_{11}$ & 367 & 309 \\
$\omega_{1}$ & .012 & .014 \\
$\lambda_{1}$ & .44 & .53 \\
$\omega_{2}$ & .0035 & .0042 \\
$\lambda_{2}$ & .32 & .38 \\
\hline
\end{tabular}

Table 6. Standard Errors of Parameter Estimates

$$
\text { OLS EGLS EGLS }
$$

\begin{tabular}{cccc} 
Parameter & NLINVC & ANOVA & MINQUE \\
\hline$\alpha$ & 418 & 382 & 413 \\
$\mathrm{~B}_{1}$ & 258 & 237 & 256 \\
$\mathrm{~B}_{2}$ & 259 & 238 & 257 \\
$\mathrm{C}_{1}$ & 222 & 204 & 221 \\
$\mathrm{M}_{1}$ & 672 & 591 & 639 \\
$(\mathrm{CM})_{\mathrm{i} i}$ & 309 & 282 & 305 \\
$\omega_{1}$ & .014 & .013 & .014 \\
$\lambda_{1}$ & .53 & .50 & .54 \\
$\omega_{2}$ & .0042 & .0040 & .0043 \\
$\lambda_{2}$ & .38 & .35 & .38 \\
\hline
\end{tabular}



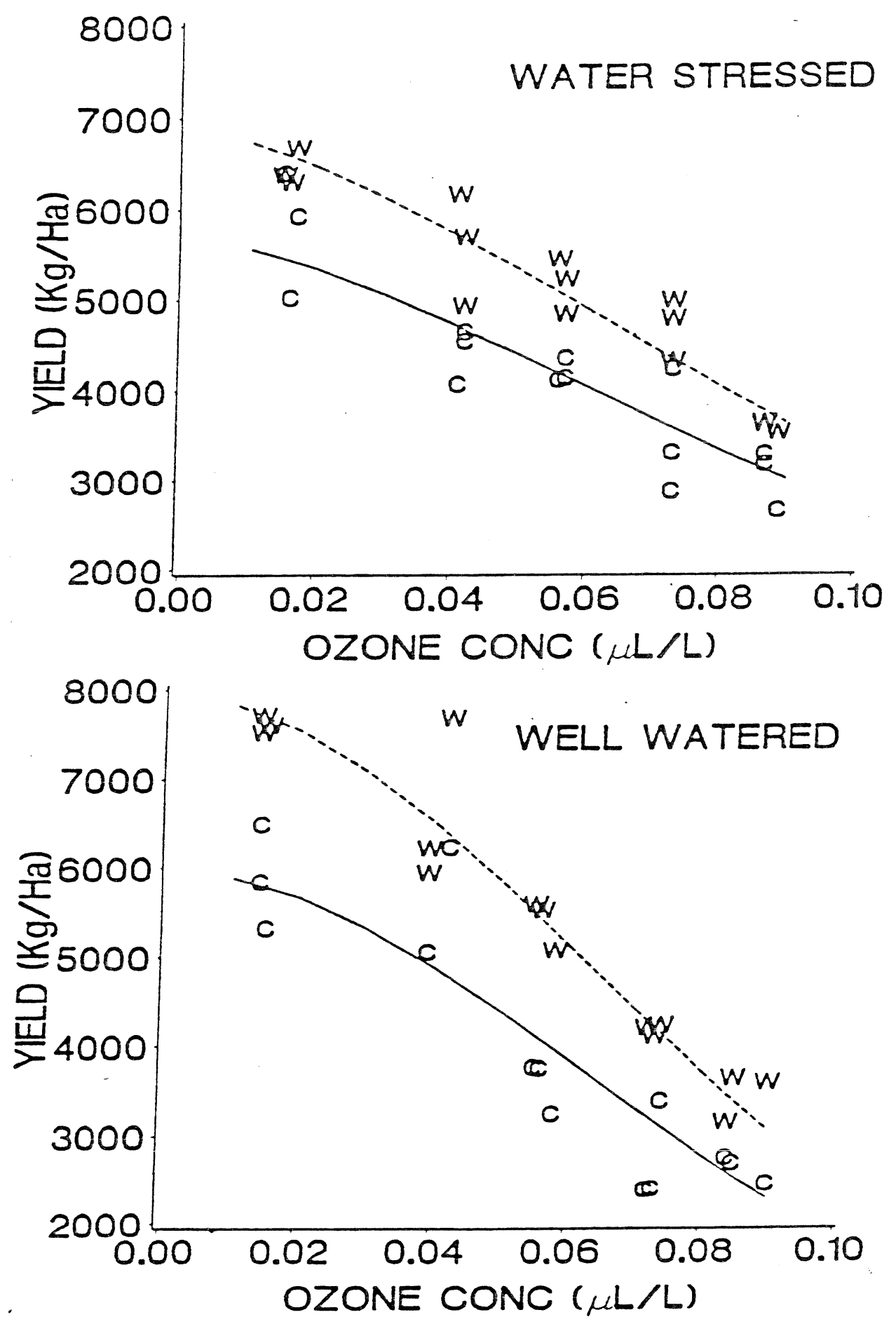

Fig. 1. Effect of ozone concentration on soybean yield. Symbol indicales cullivar: Williams or Corsoy. 